\title{
Development and Optimization of a Condition-Based Maintenance Policy with Sustainability Requirements for Production System
}

\author{
Aiping Jiang $\mathbb{D}^{1},{ }^{1}$ Ning Dong, ${ }^{1}$ Kwok Leung Tam, ${ }^{2}$ and Chonghao Lyu' ${ }^{1}$ \\ ${ }^{1}$ SHU-UTS SILC Business School, Shanghai University, 20 Chengzhong Road, Jiading District, Shanghai 201899, China \\ ${ }^{2}$ School of Mathematics and Statistics, UNSW, Sydney, NSW 2052, Australia \\ Correspondence should be addressed to Aiping Jiang; ap724@shu.edu.cn
}

Received 18 August 2017; Revised 25 November 2017; Accepted 21 December 2017; Published 28 February 2018

Academic Editor: J.-C. Cortés

Copyright (C) 2018 Aiping Jiang et al. This is an open access article distributed under the Creative Commons Attribution License, which permits unrestricted use, distribution, and reproduction in any medium, provided the original work is properly cited.

\begin{abstract}
In the field of condition-based maintenance, maintenance costs and system reliability criteria are the primary considerations for traditional maintenance management. These methods lack consideration of the environmental impact caused by equipment degradation, such as excessive emissions and energy consumption. In addition, because equipment degradation has various impacts on the ecological environment, companies with excessive emissions and energy consumption can receive huge fines, making it of great value to study ecoconscious maintenance strategies. In this paper, we propose a condition-based maintenance strategy considering energy consumption and carbon dioxide emissions. The major objective of the research is to extend a model which integrates ecological aspects with maintenance decision-making and optimization. The simulation and sensitivity analyses conducted verify that the model proposed can minimize total costs, as well as the environmental impact.
\end{abstract}

\section{Introduction}

Maintenance refers to maintaining and repairing equipment and systems to ensure they are in good condition. It is an indispensable activity related to machines, ranging from grinding or replacing a small unit to precisely moderating or repairing the whole system. When it comes to the lengthy operation of systems, such as social infrastructure, military equipment, production system, household electrical appliances, and vehicles, maintenance plays a crucial role in ensuring their safety and reliability. If the equipment and system cannot be maintained, its continuous use will lead to deterioration (e.g., aging, wear, corrosion, and creep) resulting in declining performance, reduced reliability, and even a shortened life. Within this context, condition-based maintenance strategies have been studied and widely applied by organizations as they propose an optimal maintenance policy based on the deterioration state of the system.

At present, most organizations focus solely on the relative maintenance cost when establishing a maintenance strategy for normal operation and system reliability. Minimizing cost is the primary task, while the impact of the system on the environment is seldom considered. Take, for example, a ship company that finds belt relaxation in various motor boilers, blowers, and belt conveyors or inefficiency of door adjustment in the induced draft fan, where shutdown will not happen. The company will not undertake immediate maintenance considering the cost criteria; however, its continued use will seriously affect the critical value of combustion conditions (i.e., the excess coefficient of air). Generally, when the critical value is too large, the exhaust heat loss and electricity consumption of the draft fan increases. Conversely, when the value is too small, combustion is not complete and harmful gas emissions increase, causing serious environmental pollution.

As energy and environmental issues become increasingly severe, awareness of ecological impacts is progressing in the international community. Since the late 1980s human society has been advocating a "green era," where protecting the environment, embracing nature, and promoting sustainable development are the center of all activities. In this context, green maintenance models have become the new trend 
for saving energy and resources, implementing environmental protection, and achieving sustainable development. The questions of how to achieve environmentally-friendly and efficient production and maintenance and of how to improve resource utilization rate and competitive power to create a green image have now become main concerns of many companies. In the field of maintenance management research, traditional, condition-based maintenance is mainly concerned with maintenance costs and system reliabilityoptimization, while ignoring the subsequent excess wasted gas emissions and excess energy consumption. This focus does not consider the efficient use of resources, impacts on the environment, and sustainable development. Energy, resources and the environment are all in the scope of ecology [1]; hence, studying condition-based maintenance while taking ecological factors into account can be of great value.

\section{Literature Review}

The traditional research on CBM focused on the optimization of maintenance itself but lacked consideration of ecological aspects. With the increasingly serious energy and environmental problems over the past decade, literature on ecologically aware maintenance optimization has gradually emerged. More scholars are beginning to integrate energy conservation and emission reduction into CBM management.

Van Horenbeek et al. proposed a CBM model in 2014 with the first integration of ecological factors into decisionmaking and optimization [1]. The model is an economic and ecological analysis tool that incorporates several maintenance strategies (e.g., failure-based, block-based, and use-based maintenance). Using this analysis tool, we can obtain the optimal maintenance interval considering economic, ecological, and integrated aspects. The author verified the model and validated that it can be effectively applied to the cutting tool of a turning machine. The results also reveal that the ecological optimal maintenance interval is much smaller than the economic optimal maintenance interval. Integrated cost is mainly determined by economic costs, but the ecological impact cannot be ignored. In addition, a Pareto analysis showed that, by varying the preventive maintenance interval, a slight increase in the economic cost of maintenance will significantly reduce the ecological impact.

$\mathrm{Xu}$ and Cao [2] proposed to use energy efficiency performance as an objective for maintenance by updating periodic maintenance on energy efficiency evolution, but mainly on mastering the deterioration of machine tool (a Markov process application). Shafiee et al. [3] use the deterioration level of multibladed wind turbine system to rise/trigger the unplanned maintenance action for any failure items, while electrical energy as the useful output of this system is not fully taken into account. So energy efficiency is seen only as the auxiliary result of these previous CBM plans. In addition, Mora et al. [4] point out that energy can be saved by optimal periodic preventive maintenance. Yildirim and Nezami [5] also think that energy can impact preventive maintenance by considering energy consumption in the case of minimal repair. To summarize, energy consumption in link with maintenance is mainly considered as an item to adapt a preventive maintenance plan in terms of scheduled one but not in case of CBM strategy.

Therefore, Hoang et al. [6] presented an optimization model for the CBM strategy in 2016 based on the stochastic process theory. This model assumes that the degradation increment is subject to the Gamma process and that the deterioration state, along with available output, determines the Energy Efficiency Index (EEI). If the EEI exceeds the threshold level in a periodic inspection, preventive maintenance action will be undertaken. The number of different maintenance actions in the objective function of this optimization model is affected by the EEI threshold, which fully integrates the energy consumption problem into the maintenance activity. Moreover, the total cost is divided into maintenance cost and energy consumption cost so that both of them are considered when making maintenance decisions. Compared with the traditional CBM strategy based on the deterioration of the system, the CBM strategy based on EEI proposed by the literature [6] can more efficiently reduce costs and save energy.

In addition to energy consumption, some scholars also integrate environmental degradation into the CBM strategy. Martorell et al. [7] proposed integrated multicriteria decision-making (IMCDM) in order to achieve better scores in reliability, availability, maintainability, safety, and cost $(\mathrm{RAMS}+\mathrm{C})$ at nuclear power plants. The proposed methodology integrates, among others, the risk to environment. The IMCDM is formulated as a multiobjective optimization where the decision variables are preventive maintenance interval, surveillance test interval, and maximum allowed outage time. Vassiliadis and Pistikopoulos [8] developed an optimization framework which takes into account the environmental risks and the operability characteristics at the early stage of process design. This framework permits the identification of optimal preventive maintenance schedules.

The excess of environmental damage generated by production systems is, in numerous situations, caused indirectly by the deterioration of those systems (e.g., degradation and/or corrosion yielding leakages and wear causing excess of energy consumption). For instance, in a nuclear power plant, the degradation of the mechanical shat seal of the refrigeration compressor induces toxic refrigerant leakages [9]. Yan and Hua [10] showed that the degradation of machine tools causes an increase of energy consumption which can be converted to carbon emission by referring to the standard developed by the Climate Registry.

Therefore, when it is technically possible, conditionbased monitoring, repair, and maintenance are the most appropriate activities to be adopted. It allows monitoring the degradation level and the resulting environmental damage in order to take the appropriate preventive actions and limit the risk of penalties and sometimes catastrophes. Chouikhi et al. [11] pointed out that, with the global enhancement of environmental awareness, many countries have introduced policies of severely punishing companies with excess emissions, which is overlooked by the traditional CBM strategy. 
This means the exhaust emission may have exceeded the standard before maintenance. In order to remedy this problem, Chouikhi et al. proposed a modeling method of CBM in 2012 based on probabilistic statistical theory to integrate environmental issues into maintenance actions. The two parameters (the time interval from equipment in good condition to the time point at which the exhaust gas leaks go beyond the warning line, in addition to the time interval from exceeding the warning line to the downtime) are assumed to be subject to a joint probability density function, so then the probability of preventive maintenance (when the leak exceeds the critical line), corrective maintenance, and inspection can be obtained; thus, we get the expected total cost per time unit during the renewal cycle.

Tlili et al. [12] made some improvement on the method [11] and proposed a threshold lower than the critical level fixed by legislation as a criterion for judging whether to perform preventive maintenance, thereby avoiding the huge amount of economic penalties for excess exhaust gas. Furthermore, Tlili et al. [12] integrated the penalty cost for excess emissions into the cost function, making the total cost in the objective function more comprehensive. In addition, they compared periodic inspection with nonperiodic inspection, with the results showing that the nonperiodic inspection policy can save more costs and induce less environmental pollution.

Traditional CBM does not take into account the environmental attributes of maintenance activities. In view of this problem, although some scholars integrate environmental issues into CBM strategy, the factors involved are not comprehensive. The literature [11, 12] quantified the mutual effects of exhaust emissions and maintenance activities but was limited to the emissions. In reality, maintenance activities also induce energy consumption, so the decision optimization should also consider this ecological factor to reflect how maintenance activities and ecology impact each other. This paper proposes a CBM strategy that combines energy consumption and $\mathrm{CO} 2$ emissions with the deterioration state of the system, establishing a single-unit system maintenance optimization model based on the probabilistic characteristics of the combination of energy consumption and $\mathrm{CO} 2$ emissions. The model makes maintenance decisions based on the threshold of these two ecological factors; in this way, the ecofactors are integrated into maintenance activities, enabling the maintenance activities both to save economic costs and to effectively conserve energy and protect the environment, which can ultimately achieve the goal of sustainable development.

\section{Integrative Condition-Based Maintenance Model for Single-Unit Systems Based on Sustainability Requirements}

\subsection{Problem Description and Model Assumptions}

3.1.1. Problem Description. This paper considers the ecological impact of a production system assimilated to a single-unit system as it continuously degrades; that is, it considers excessive carbon dioxide emissions and excessive energy consumption. Since in many cases environmental degradation generated by the production system has a direct (or indirect) relationship with the system degradation, we can study carbon dioxide emissions and energy consumption in replacement of the deterioration state of the system [11]. It is assumed that the system's carbon dioxide emissions and energy consumption are, respectively, subject to a Wiener process and that the degradation level of the system can only be known through inspection. This paper concerns a system that is periodically inspected. If inspection results show that any one of the system's $\mathrm{CO} 2$ emissions or energy consumption exceeds the critical value, $U_{i}\left(U_{1}\right.$ refers to the critical level of $\mathrm{CO} 2$ emissions fixed by legislation and $U_{2}$ refers to the critical level of energy consumption fixed by legislation), the enterprise has to pay a heavy penalty. Meanwhile, a tremendous impact on the environment will be averted, along with a waste of resources, as when a CM action must be performed. In order to lower the possibility of getting into such an unfavorable situation, a threshold level $L_{i}$ lower than $U_{i}$ is set to trigger a PM action $\left(L_{1}\right.$ refers to PM threshold for $\mathrm{CO} 2$ emission and $L_{2}$ refers to PM threshold for energy consumption). In case an inspection shows that any of the environmental indicators exceeds the PM threshold, a PM action should be undertaken; otherwise, no maintenance action will be taken before the next inspection.

3.1.2. Model Assumptions. Notations section explains the notations related to the model of the CBM strategy considering carbon dioxide emissions and energy consumption, for ease of understanding.

For the proposed model, the following assumptions are made.

(1) $\mathrm{CO} 2$ emissions and energy consumption of the system are subject to two independent Wiener processes.

(2) The time of CO2 emissions and energy consumption from the moment when the degradation hits the PM threshold level $L$ to the instant at which it reaches the critical level are subject to two independent IG processes.

(3) The inspection is periodic and the period is $\tau=$ $k \Delta t, k=1,2,3, \ldots$, where $\Delta t$ is unit of time.

(4) The inspection is perfect and time spent is negligible.

(5) The actions of preventive maintenance and corrective maintenance return the equipment to its original state and the maintenance time is negligible.

(6) According to the inspection results, only one of the three following events is possible: undertake a PM action, undertake a CM action, or do nothing before next inspection.

(7) All maintenance costs, inspection costs, and penalty costs are included in the average cost and assumed to be constants.

(8) A renewal cycle is from current time to the end of maintenance. 
(9) The time from current time to the instant at which the degradation hits the PM threshold and the time from the instant at which it reaches the critical level follow independent normal distributions.

3.2. Model Establishment. Tlili et al. [12] assume the environmental degradation process of the equipment (namely, the carbon dioxide emission of the equipment) obeys a Wiener process:

$$
X(t)=\lambda t+\sigma W(t), \quad t>0
$$

where $X(t)$ denotes standard Brownian motion, $\lambda$ is the drift coefficient, $\sigma$ is the diffusion coefficient, and the mean and variance $X(t)$ are expressed by $\mu t$ and $\sigma^{2} t$, respectively. $X(t)$ has the following properties:

(a) $X(0)=0$.

(b) For any time sequence $t_{i}, i=1,2,3, \ldots, n(0<$ $\left.t_{1}<t_{2}<\cdots<t_{n}\right)$, the random increments $X\left(t_{1}\right), X\left(t_{2}-\right.$ $\left.t_{1}\right), \ldots, X\left(t_{n}-t_{n-1}\right)$, where $X\left(t_{j}-t_{j-1}\right)=X\left(t_{j}\right)-X\left(t_{j-1}\right)$, are independent, and any $X(t)-X(r), t>0, r>0$ follows a normal distribution $N\left(0, \sigma^{2}|t-r|\right)$.

(c) The paths of $X(t)$ are continuous with probability one.

The time $T_{U}$ from current time to the moment when the degradation reaches the critical level, $U$, follows the inverse Gaussian distribution and can be defined as

$$
T_{U}=\inf \{t \geq 0 \mid X(t) \geq U\}
$$

The pdf of $T_{U}$ is expressed as

$$
f_{T_{U}}(t)=\frac{U}{\sqrt{2 \pi t^{3} \sigma^{2}}} \exp \left(-\frac{(U-\lambda t)^{2}}{2 \sigma^{2} t}\right)
$$

and the cdf of $T_{U}$ is given by

$$
\begin{aligned}
F_{T_{U}}(t)= & \Phi\left(\frac{-U+\lambda t}{\sigma \sqrt{t}}\right) \\
& +\exp \left(\frac{2 \lambda U}{\sigma^{2}}\right) \Phi\left(\frac{-U-\lambda t}{\sigma \sqrt{t}}\right) .
\end{aligned}
$$

The time $T_{S}$ from the instant at which the degradation hits the PM threshold level $L$ to the moment when it reaches the critical level, $U$, can be defined as

$$
\Delta T_{S}=T_{U}-T_{L}=\inf \{s \geq 0 \mid X(t+s) \geq U\} .
$$

If $L<U$, otherwise $\Delta T_{S}=0$.

The degradation increments are independent based on property (b) of the Wiener process; therefore, if $L<U, T_{S}$ can be expressed as follows:

$$
\begin{aligned}
T_{S} & =T_{U}-T_{L}=\inf \{s \geq 0 \mid X(t+s) \geq U\} \\
& =\inf \{s \geq 0 \mid X(t+s) \geq U-L\} .
\end{aligned}
$$

The pdf of $T_{S}$ is written as follows:

$$
f_{T_{U}-T_{L}}(t)=\frac{U-L}{\sqrt{2 \pi t^{3} \sigma^{2}}} \exp \left(-\frac{(U-L-\lambda t)^{2}}{2 \sigma^{2} t}\right) .
$$

And the cdf of $T_{S}$ is given by

$$
\begin{aligned}
& F_{T_{U}-T_{L}}(t) \\
& =\Phi\left(\frac{-(U-L)+\lambda t}{\sigma \sqrt{t}}\right) \\
& \quad+\exp \left(\frac{2 \lambda(U-L)}{\sigma^{2}}\right) \Phi\left(\frac{-(U-L)-\lambda t}{\sigma \sqrt{t}}\right) .
\end{aligned}
$$

This paper will take into account energy consumption on the basis of Tlili et al's literature. Assuming the CO2 emissions and energy consumption of the system follow two independent Wiener processes, the threshold level of CO2 emissions and energy consumption are $L_{1}$ and $L_{2}$, respectively, and the critical level of $\mathrm{CO} 2$ emissions and energy consumption fixed by legislation are $U_{1}$ and $U_{2}$, respectively.

The Wiener process can be expressed as

$$
X_{i}(t)=\lambda_{i} t+\sigma_{i} W(t), \quad t>0,
$$

where $X(t)$ denotes standard Brownian motion, $\lambda_{i}$ is the drift coefficient, $\sigma_{i}$ is the diffusion coefficient, and the mean and variance of $X(t)$ are expressed as $\mu t$ and $\sigma^{2} t$, respectively.

The time $T_{U_{i}}$ from current time to the instant at which the degradation hits the critical level, $U_{i}$, follows the inverse Gaussian distribution and can be defined as

$$
T_{U_{i}}=\inf \left\{t \geq 0 \mid X_{i}(t) \geq U_{i}\right\}, \quad i=1,2
$$

The pdf of $T_{U_{i}}$ is expressed as

$$
f_{T_{U_{i}}}(t)=\frac{U_{i}}{\sqrt{2 \pi t^{3} \sigma_{i}^{2}}} \exp \left(-\frac{\left(U_{i}-\lambda_{i} t\right)^{2}}{2 \sigma_{i}^{2} t}\right), \quad i=1,2 .
$$

And the cdf of $T_{U_{i}}$ is given by

$$
\begin{aligned}
F_{T_{U_{i}}}(t)= & \Phi\left(\frac{-U_{i}+\lambda_{i} t}{\sigma_{i} \sqrt{t}}\right) \\
& +\exp \left(\frac{2 \lambda_{i} U_{i}}{\sigma_{i}^{2}}\right) \Phi\left(\frac{-U_{i}-\lambda_{i} t}{\sigma_{i} \sqrt{t}}\right) .
\end{aligned}
$$

The time $T_{S_{i}}$ from the instant at which the degradation hits the PM threshold level $L$ to the moment when it reaches the critical level $U$ can be defined as 


$$
T_{S_{i}}=T_{U_{i}}-T_{L_{i}}=\inf \left\{s_{i} \geq 0 \mid X\left(t+s_{i}\right) \geq U_{i}\right\} .
$$

If $L_{i}<U_{i}$, otherwise $T_{S_{i}}=0$.

Based on property (b) of the Wiener process, the degradation increments are independent; hence, if $L<U, T_{S_{i}}$ can be written as follows:

$$
\begin{aligned}
T_{S_{i}} & =T_{U_{i}}-T_{L_{i}}=\inf \left\{S_{i} \geq 0 \mid X\left(t+S_{i}\right) \geq U_{i}\right\} \\
& =\inf \left\{S_{i} \geq 0 \mid X\left(t+S_{i}\right) \geq U_{i}-L_{i}\right\}, \quad i=1,2 .
\end{aligned}
$$

The pdf of $T_{S_{i}}$ is expressed as

$$
f_{T_{U_{i}}-T_{L_{i}}}(t)=\frac{U_{i}-L_{i}}{\sqrt{2 \pi t^{3} \sigma_{i}^{2}}} \exp \left(-\frac{\left(U_{i}-L_{i}-\lambda_{i} t\right)^{2}}{2 \sigma_{i}^{2} t}\right) .
$$

And the cdf of $T_{S_{i}}$ is given by

$$
\begin{aligned}
F_{T_{U_{i}}-T_{L_{i}}} & (t) \\
= & \Phi\left(\frac{-\left(U_{i}-L_{i}\right)+\lambda_{i} t}{\sigma_{i} \sqrt{t}}\right) \\
& +\exp \left(\frac{2 \lambda_{i}\left(U_{i}-L_{i}\right)}{\sigma_{i}^{2}}\right) \Phi\left(\frac{-\left(U_{i}-L_{i}\right)-\lambda_{i} t}{\sigma_{i} \sqrt{t}}\right) .
\end{aligned}
$$

We suppose that periodic inspections are conducted at times $i \pi, i=1,2,3, \ldots$. The objective is to determine the optimal PM threshold level of $\mathrm{CO} 2$ emission $L_{1}^{*}$, optimal PM threshold level of energy consumption $L_{2}^{*}$, and the interinspection period $\tau^{*}$.
According to classical renewal theory, the average longrun cost rate per time unit can be expressed over a renewal cycle $S$, defined as the interval between successive maintenance actions.

Hence, the expression of the average long-run cost rate is given by

$$
E C\left(L_{1}, L_{2}, \tau\right)=\frac{E\left[C_{T}\left(L_{1}, L_{2}, \tau\right)\right]}{E\left[C_{\text {cycle }}\left(L_{1}, L_{2}, \tau\right)\right]}
$$

where $E\left[C_{T}\right]$ denotes the expected cost during the cycle and $E\left[C_{\text {cycle }}\right]$ denotes the expected renewal cycle length.

3.2.1. The Expected Renewal Cycle Length. Suppose the renewal period of the system denotes $T=i \pi, i=$ $1,2,3, \ldots$. Since the system is considered to be as good as new after both maintenance actions (PM or CM), there are three possible events determined by the system's $\mathrm{CO} 2$ emissions and energy consumption, which are now described.

(a) The time $T_{L_{1}}$ from current time to the instant at which the CO2 emission $X_{1}(t)$ reaches the PM threshold level is found $(i-1) \tau<T_{L_{1}} \leq i \tau$, while the time $T_{L_{2}}$ from current time to the instant at which the energy consumption $X_{2}(t)$ reaches the PM threshold level is found $T_{L_{2}} \geq i \tau$. At $i \tau, \mathrm{PM}$ or $\mathrm{CM}$ action is performed since the measured $\mathrm{CO} 2$ emission $X_{1}(t)$ has exceeded the PM threshold level $L_{1}$. Express this event as $A_{1}(i, \tau)=\left\{(i-1) \tau<T_{L_{1}} \leq i \tau, T_{L_{2}} \geq i \tau\right\}$, where $i=1,2,3, \ldots$, and its probability of occurrence is given by

$$
\begin{aligned}
P\left(A_{1}(i, \tau)\right) & =\int_{(i-1) \tau}^{i \tau} \int_{i \tau}^{\infty} F(u, v) d u d v=\int_{(i-1) \tau}^{i \tau} \int_{i \tau}^{\infty} f_{T_{L_{1}}}(u) f_{T_{L_{2}}}(v) d u d v \\
& =\int_{(i-1) \tau}^{i \tau} \frac{L_{1}}{\sqrt{2 \pi u^{3} \sigma_{1}^{2}}} \exp \left(-\frac{\left(L_{1}-\lambda_{1} u\right)^{2}}{2 \sigma_{1}^{2} u}\right) d u \int_{i \tau}^{\infty} \frac{L_{2}}{\sqrt{2 \pi v^{3} \sigma_{2}^{2}}} \exp \left(-\frac{\left(L_{2}-\lambda_{2} v\right)^{2}}{2 \sigma_{2}^{2} v}\right) d v .
\end{aligned}
$$

(b) The time $T_{L_{2}}$ from current time to the instant at which the energy consumption $X_{2}(t)$ reaches the PM threshold is found, $(i-1) \tau<T_{L_{2}} \leq i \tau$, while the time $T_{L_{1}}$ from current time to the instant at which the $\mathrm{CO} 2$ emission $X_{1}(t)$ reaches the PM threshold is found, $T_{L_{1}} \geq i \tau$. At $i \tau, \mathrm{PM}$ or $\mathrm{CM}$ action is performed since the measured energy consumption $X_{2}(t)$ has exceeded the PM threshold level $L_{2}$. Express this event as $A_{2}(i, \tau)=\left\{(i-1) \tau<T_{L_{2}} \leq i \tau, T_{L_{1}} \geq i \tau\right\}$, where $i=1,2,3, \ldots$, and its probability of occurrence is given by

$$
\begin{aligned}
P\left(A_{2}(i, \tau)\right) & =\int_{i \tau}^{\infty} \int_{(i-1) \tau}^{i \tau} F(u, v) d u d v=\int_{i \tau}^{\infty} \int_{(i-1) \tau}^{i \tau} f_{T_{L_{1}}}(u) f_{T_{L_{2}}}(v) d u d v \\
& =\int_{i \tau}^{\infty} \frac{L_{1}}{\sqrt{2 \pi u^{3} \sigma_{1}^{2}}} \exp \left(-\frac{\left(L_{1}-\lambda_{1} u\right)^{2}}{2 \sigma_{1}^{2} u}\right) d u \int_{(i-1) \tau}^{i \tau} \frac{L_{2}}{\sqrt{2 \pi v^{3} \sigma_{2}^{2}}} \exp \left(-\frac{\left(L_{2}-\lambda_{2} v\right)^{2}}{2 \sigma_{2}^{2} v}\right) d v .
\end{aligned}
$$


(c) The time from current time to the instant at which $X_{1}(t)$ and $X_{2}(t)$ reaches the threshold level are found $(i-1) \tau<$ $T_{L_{1}} \leq i \tau$ and $(i-1) \tau<T_{L_{2}} \leq i \tau$, respectively. At $i \tau$, PM or CM action is performed since both $X_{1}(t)$ and $X_{2}(t)$ have exceeded the PM threshold level $L_{1}$.
Express this event as $A_{3}(i, \tau)=\left\{(i-1) \tau<T_{L_{1}} \leq i \tau,(i-\right.$ 1) $\left.\tau<T_{L_{2}} \leq i \tau\right\}$, where $i=1,2,3, \ldots$, and its probability of occurrence is given by

$$
\begin{aligned}
P\left(A_{3}(i, \tau)\right) & =\int_{(i-1) \tau}^{i \tau} \int_{(i-1) \tau}^{i \tau} F(u, v) d u d v=\int_{(i-1) \tau}^{i \tau} \int_{(i-1) \tau}^{i \tau} f_{T_{L_{1}}}(u) f_{T_{L_{2}}}(v) d u d v \\
& =\int_{(i-1) \tau}^{i \tau} \frac{L_{1}}{\sqrt{2 \pi u^{3} \sigma_{1}^{2}}} \exp \left(-\frac{\left(L_{1}-\lambda_{1} u\right)^{2}}{2 \sigma_{1}^{2} u}\right) d u \int_{(i-1) \tau}^{i \tau} \frac{L_{2}}{\sqrt{2 \pi v^{3} \sigma_{2}^{2}}} \exp \left(-\frac{\left(L_{2}-\lambda_{2} v\right)^{2}}{2 \sigma_{2}^{2} v}\right) d v
\end{aligned}
$$

Finally, the expected renewal cycle length is expressed as

$$
\begin{aligned}
E\left[C_{\text {cycle }}\left(L_{1}, L_{2}, \tau\right)\right]= & \sum_{i=1}^{\infty}\left(P\left(A_{1}(i, \tau)\right)+P\left(A_{2}(i, \tau)\right)+P\left(A_{3}(i, \tau)\right)\right) \\
= & \int_{(i-1) \tau}^{i \tau} \frac{L_{1}}{\sqrt{2 \pi u^{3} \sigma_{1}^{2}}} \exp \left(-\frac{\left(L_{1}-\lambda_{1} u\right)^{2}}{2 \sigma_{1}^{2} u}\right) d u \int_{i \tau}^{\infty} \frac{L_{2}}{\sqrt{2 \pi v^{3} \sigma_{2}^{2}}} \exp \left(-\frac{\left(L_{2}-\lambda_{2} v\right)^{2}}{2 \sigma_{2}^{2} v}\right) d v \\
& +\int_{i \tau}^{\infty} \frac{L_{1}}{\sqrt{2 \pi u^{3} \sigma_{1}^{2}}} \exp \left(-\frac{\left(L_{1}-\lambda_{1} u\right)^{2}}{2 \sigma_{1}^{2} u}\right) d u \int_{(i-1) \tau}^{i \tau} \frac{L_{2}}{\sqrt{2 \pi v^{3} \sigma_{2}^{2}}} \exp \left(-\frac{\left(L_{2}-\lambda_{2} v\right)^{2}}{2 \sigma_{2}^{2} v}\right) d v \\
& +\int_{(i-1) \tau}^{i \tau} \frac{L_{1}}{\sqrt{2 \pi u^{3} \sigma_{1}^{2}}} \exp \left(-\frac{\left(L_{1}-\lambda_{1} u\right)^{2}}{2 \sigma_{1}^{2} u}\right) d u \int_{(i-1) \tau}^{i \tau} \frac{L_{2}}{\sqrt{2 \pi v^{3} \sigma_{2}^{2}}} \exp \left(-\frac{\left(L_{2}-\lambda_{2} v\right)^{2}}{2 \sigma_{2}^{2} v}\right) d v .
\end{aligned}
$$

3.2.2. Expected Total Cost within a Cycle. The expected total cost within a cycle can be expressed as

$$
\begin{aligned}
E\left[\left(L_{1}, L_{2}, \tau\right)\right]= & C_{C} P_{P}+C_{P} P_{P}+C_{I} E[N] \\
& +C_{\mathrm{CO}_{2}} E\left[T_{\xi}\right]+C_{\text {energy }} E\left[T_{\delta}\right] .
\end{aligned}
$$

The three first elements in the formula are PM, CM, and the inspection average costs within a cycle, respectively. $C_{\mathrm{CO}_{2}} E\left[T_{\xi}\right]$ denotes the penalty cost for excess $\mathrm{CO} 2$ emissions within a cycle and $C_{\text {energy }} E\left[T_{\delta}\right]$ denotes the penalty cost for excess energy consumption within a cycle.

(1) The Probability $P_{P}$ That the Cycle Ends with a Preventive Maintenance Action. Suppose a PM action is undertaken in the system at $i \tau$. There are three possible events, as follows.

(a) At $i \tau$, the $\mathrm{CO} 2$ emission $X_{1}(t)$ was higher than the PM threshold level $L_{1}$, but lower than the critical level $U_{1}$, while the energy consumption $X_{2}(t)$ has not reached the PM threshold level $L_{2}$. Express this event as $B_{1}(i, \tau)=\{(i-1) \tau<$ $\left.T_{L_{1}} \leq i \tau<T_{U_{1}}, T_{L_{2}} \geq i \tau\right\}$, then its probability of occurrence is given by

$$
\begin{aligned}
P & \left(B_{1}(i, \tau)\right)=\int_{(i-1) \tau}^{i \tau} \int_{i \tau-u}^{\infty} \int_{i \tau}^{\infty} F(u, s, v) d u d s d v \\
& =\int_{(i-1) \tau}^{i \tau} \int_{i \tau-u}^{\infty} \int_{i \tau}^{\infty} f_{T_{L_{1}}}(u) f_{T_{U_{1}}-T_{L_{1}}}(s) f_{T_{L_{2}}}(v) d u d s d v \\
& =\int_{(i-1) \tau}^{i \tau} \int_{i \tau-u}^{\infty} f_{T_{L_{1}}}(u) \\
& \cdot f_{T_{U_{1}}-T_{L_{1}}}(s) d u d s \int_{i \tau}^{\infty} f_{T_{L_{2}}}(v) d v \\
& =\int_{(i-1) \tau}^{i \tau} f_{T_{L_{1}}}(u)\left[1-F_{T_{U_{1}}-T_{L_{1}}}(i \tau\right. \\
& -u)] d u \int_{i \tau}^{\infty} f_{T_{L_{2}}}(v) d v \\
& =\int_{(i-1) \tau}^{i \tau} \frac{L_{1}}{\sqrt{2 \pi u^{3} \sigma_{1}^{2}}} \exp \left(-\frac{\left(L_{1}-\lambda_{1} u\right)^{2}}{2 \sigma_{1}^{2} u}\right)[1 \\
& -\Phi\left(\frac{-\left(U_{1}-L_{1}\right)+\lambda_{1} u}{\sigma_{1} \sqrt{u}}\right)-\exp \left(\frac{2 \lambda_{1}\left(U_{1}-L_{1}\right)}{\sigma_{1}^{2}}\right) \\
& \left.\cdot \Phi\left(\frac{-\left(U_{1}-L_{1}\right)-\lambda_{1} u}{\sigma_{1} \sqrt{u}}\right)\right] d u \int_{i \tau}^{\infty} \frac{L_{2}}{\sqrt{2 \pi v^{3} \sigma_{2}^{2}}} \\
& \cdot \exp \left(-\frac{\left(L_{2}-\lambda_{2} v\right)^{2}}{2 \sigma_{2}^{2} v}\right) d v .
\end{aligned}
$$


(b) At $i \tau$, the energy consumption $X_{2}(t)$ was higher than the PM threshold level $L_{2}$, but lower than the critical level $U_{2}$, while the CO2 emissions $X_{1}(t)$ has not reached the PM threshold level $L_{1}$. Express this event as $B_{2}(i, \tau)=\{(i-1) \tau<$ $\left.T_{L_{2}} \leq i \tau<T_{U_{2}}, T_{L_{1}} \geq i \tau\right\}$, then its probability of occurrence is given by

$$
\begin{aligned}
& P\left(B_{2}(i, \tau)\right)=\int_{i \tau}^{\infty} \int_{(i-1) \tau}^{i \tau} \int_{i \tau-v}^{\infty} F(u, t, v) d u d v d t=\int_{i \tau}^{\infty} \int_{(i-1) \tau}^{i \tau} \int_{i \tau-v}^{\infty} f_{T_{L_{1}}}(u) f_{T_{L_{2}}}(v) f_{T_{U_{2}}-T_{L_{2}}}(t) d u d v d t \\
& \quad=\int_{i \tau}^{\infty} f_{T_{L_{1}}}(u) d u \int_{(i-1) \tau}^{i \tau} \int_{i \tau-v}^{\infty} f_{T_{L_{2}}}(v) f_{T_{U_{2}}-T_{L_{2}}}(t) d v d t \\
& \quad=\int_{i \tau}^{\infty} f_{T_{L_{1}}}(u) d u \int_{(i-1) \tau}^{i \tau} f_{T_{L_{2}}}(v)\left[1-F_{T_{U_{2}}-T_{L_{2}}}(i \tau-v)\right] d v \\
& \quad=\int_{i \tau}^{\infty} \frac{L_{1}}{\sqrt{2 \pi u^{3} \sigma_{1}^{2}}} \exp \left(-\frac{\left(L_{1}-\lambda_{1} u\right)^{2}}{2 \sigma_{1}^{2} u}\right) d u \cdot \int_{(i-1) \tau}^{i \tau} \frac{L_{2}}{\sqrt{2 \pi u^{3} \sigma_{2}^{2}}} \exp \left(-\frac{\left(L_{2}-\lambda_{2} v\right)^{2}}{2 \sigma_{2}^{2} v}\right) \cdot[1 \\
& \left.\quad-\Phi\left(\frac{-\left(U_{2}-L_{2}\right)+\lambda_{2} v}{\sigma_{1} \sqrt{v}}\right)-\exp \left(\frac{2 \lambda_{2}\left(U_{2}-L_{2}\right)}{\sigma_{2}^{2}}\right) \Phi\left(\frac{-\left(U_{2}-L_{2}\right)-\lambda_{2} v}{\sigma_{2} \sqrt{v}}\right)\right] d v .
\end{aligned}
$$

(c) At i , both $X_{1}(t)$ and $X_{2}(t)$ were higher than the PM threshold level $L_{i}$, but lower than the critical value $U_{i}$. Express this event as $B_{3}(i, \tau)=\left\{(i-1) \tau<T_{L_{1}} \leq i \tau<T_{U_{1}},(i-1) \tau<\right.$ $\left.T_{L_{2}} \leq i \tau<T_{U_{2}}\right\}$, then its probability of occurrence is given by

$$
\begin{aligned}
& P\left(B_{3}(i, \tau)\right)=\int_{(i-1) \tau}^{i \tau} \int_{i \tau-u}^{\infty} \int_{(i-1) \tau}^{i \tau} \int_{i \tau-v}^{\infty} F(u, s, v, t) d u d s d v d t \\
& =\int_{(i-1) \tau}^{i \tau} \int_{i \tau-u}^{\infty} \int_{(i-1) \tau}^{i \tau} \int_{i \tau-v}^{\infty} f_{T_{L_{1}}}(u) f_{T_{U_{1}}-T_{L_{1}}}(s) f_{T_{L_{2}}}(v) f_{T_{U_{2}}-T_{L_{2}}}(t) d u d s d v d t=\int_{(i-1) \tau}^{i \tau} \int_{i \tau-u}^{\infty} f_{T_{L_{1}}}(u) \\
& \cdot f_{T_{U_{1}}-T_{L_{1}}}(s) d u d s \int_{(i-1) \tau}^{i \tau} \int_{i \tau-v}^{\infty} f_{T_{L_{2}}}(v) f_{T_{U_{2}}-T_{L_{2}}}(t) d v d t=\int_{(i-1) \tau}^{i \tau} f_{T_{L_{1}}}(u) \\
& \cdot\left[1-F_{T_{U_{1}}-T_{L_{1}}}(i \tau-u)\right] d u \cdot \int_{(i-1) \tau}^{i \tau} f_{T_{L_{2}}}(v)\left[1-F_{T_{U_{2}}-T_{L_{2}}}(i \tau-v)\right] d v=\int_{(i-1) \tau}^{i \tau} \frac{L_{1}}{\sqrt{2 \pi u^{3} \sigma_{1}^{2}}} \exp \left(-\frac{\left(L_{1}-\lambda_{1} u\right)^{2}}{2 \sigma_{1}^{2} u}\right) \\
& \cdot\left[1-\Phi\left(\frac{-\left(U_{1}-L_{1}\right)+\lambda_{1} u}{\sigma_{1} \sqrt{u}}\right)-\exp \left(\frac{2 \lambda_{1}\left(U_{1}-L_{1}\right)}{\sigma_{1}^{2}}\right) \Phi\left(\frac{-\left(U_{1}-L_{1}\right)-\lambda_{1} u}{\sigma_{1} \sqrt{u}}\right)\right] d u \\
& \cdot \int_{(i-1) \tau}^{i \tau} \frac{L_{2}}{\sqrt{2 \pi u^{3} \sigma_{2}^{2}}} \exp \left(-\frac{\left(L_{2}-\lambda_{2} v\right)^{2}}{2 \sigma_{2}^{2} v}\right) \cdot\left[1-\Phi\left(\frac{-\left(U_{2}-L_{2}\right)+\lambda_{2} v}{\sigma_{1} \sqrt{v}}\right)-\exp \left(\frac{2 \lambda_{2}\left(U_{2}-L_{2}\right)}{\sigma_{2}^{2}}\right)\right. \\
& \left.\quad \Phi\left(\frac{-\left(U_{2}-L_{2}\right)-\lambda_{2} v}{\sigma_{2} \sqrt{v}}\right)\right] d v .
\end{aligned}
$$


Therefore, the probability $P_{P}$ that the cycle ends with a preventive maintenance action is expressed as

$$
\begin{aligned}
& P_{P}\left(L_{1}, L_{2}, \tau\right)=\sum_{i=1}^{\infty}\left[P\left(B_{1}(i, \tau)\right)+P\left(B_{2}(i, \tau)\right)+P\left(B_{3}(i, \tau)\right)\right]=\sum_{i=1}^{\infty}\left\{\int_{(i-1) \tau}^{i \tau} \frac{L_{1}}{\sqrt{2 \pi u^{3} \sigma_{1}^{2}}} \exp \left(-\frac{\left(L_{1}-\lambda_{1} u\right)^{2}}{2 \sigma_{1}^{2} u}\right)\right. \\
& \cdot\left[1-\Phi\left(\frac{-\left(U_{1}-L_{1}\right)+\lambda_{1} u}{\sigma_{1} \sqrt{u}}\right)-\exp \left(\frac{2 \lambda_{1}\left(U_{1}-L_{1}\right)}{\sigma_{1}^{2}}\right) \Phi\left(\frac{-\left(U_{1}-L_{1}\right)-\lambda_{1} u}{\sigma_{1} \sqrt{u}}\right)\right] d u \int_{i \tau}^{\infty} \frac{L_{2}}{\sqrt{2 \pi v^{3} \sigma_{2}^{2}}} \\
& \cdot \exp \left(-\frac{\left(L_{2}-\lambda_{2} v\right)^{2}}{2 \sigma_{2}^{2} v}\right) d v+\int_{i \tau}^{\infty} \frac{L_{1}}{\sqrt{2 \pi u^{3} \sigma_{1}^{2}}} \exp \left(-\frac{\left(L_{1}-\lambda_{1} u\right)^{2}}{2 \sigma_{1}^{2} u}\right) d u \int_{(i-1) \tau}^{i \tau} \frac{L_{2}}{\sqrt{2 \pi u^{3} \sigma_{2}^{2}}} \exp \left(-\frac{\left(L_{2}-\lambda_{2} v\right)^{2}}{2 \sigma_{2}^{2} v}\right) \\
& \cdot\left[1-\Phi\left(\frac{-\left(U_{2}-L_{2}\right)+\lambda_{2} v}{\sigma_{1} \sqrt{v}}\right)-\exp \left(\frac{2 \lambda_{2}\left(U_{2}-L_{2}\right)}{\sigma_{2}^{2}}\right) \Phi\left(\frac{-\left(U_{2}-L_{2}\right)-\lambda_{2} v}{\sigma_{2} \sqrt{v}}\right)\right] d v \\
& +\int_{(i-1) \tau}^{i \tau} \frac{L_{1}}{\sqrt{2 \pi u^{3} \sigma_{1}^{2}}} \exp \left(-\frac{\left(L_{1}-\lambda_{1} u\right)^{2}}{2 \sigma_{1}^{2} u}\right) \\
& \cdot\left[1-\Phi\left(\frac{-\left(U_{1}-L_{1}\right)+\lambda_{1} u}{\sigma_{1} \sqrt{u}}\right)-\exp \left(\frac{2 \lambda_{1}\left(U_{1}-L_{1}\right)}{\sigma_{1}^{2}}\right) \Phi\left(\frac{-\left(U_{1}-L_{1}\right)-\lambda_{1} u}{\sigma_{1} \sqrt{u}}\right)\right] d u \\
& \int_{(i-1) \tau}^{i \tau} \frac{L_{2}}{\sqrt{2 \pi u^{3} \sigma_{2}^{2}}} \exp \left(-\frac{\left(L_{2}-\lambda_{2} v\right)^{2}}{2 \sigma_{2}^{2} v}\right) \\
& \left.\cdot\left[1-\Phi\left(\frac{-\left(U_{2}-L_{2}\right)+\lambda_{2} v}{\sigma_{1} \sqrt{v}}\right)-\exp \left(\frac{2 \lambda_{2}\left(U_{2}-L_{2}\right)}{\sigma_{2}^{2}}\right) \Phi\left(\frac{-\left(U_{2}-L_{2}\right)-\lambda_{2} v}{\sigma_{2} \sqrt{v}}\right)\right] d v\right\} \text {. }
\end{aligned}
$$

The sum of $P_{P}$ and $P_{C}$ equals 1 as follows:

$$
P_{P}+P_{C}=1
$$

Probability $P_{C}$ that the cycle ends with a corrective maintenance action is

$$
P_{C}\left(L_{1}, L_{2}, \tau\right)=1-P_{P}\left(L_{1}, L_{2}, \tau\right) \text {. }
$$

(2) The Expected Number of Inspections during a Cycle. The expected renewal cycle length can be obtained from (1), which is here considered to be the interval between consecutive maintenance actions. It is assumed that the maintenance action to be performed is determined after the inspection using $\mathrm{CO} 2$ emissions or energy consumption and that the maintenance time is negligible. Since the inspection is periodic and the time interval is $\tau$, while the renewal period is an integral multiple of $\tau$, the expected number of inspections during a cycle can be expressed by

$$
E[N]=\frac{E_{\text {cycle }}\left[\left(L_{1}, L_{2}, \tau\right)\right]}{\tau} .
$$

(3) The Average Time for Paying a Penalty during a Cycle. At $i \tau$, in case an inspection result shows that $\mathrm{CO} 2$ emissions or energy consumption has exceeded the critical level, the company has to pay a heavy penalty for the excess amount. The discussion here is divided into two circumstances.

(a) The Average Time for Generation of an Excess Amount of CO2 Emissions. At $i \tau, \mathrm{CO} 2$ emissions $X_{1}(t)$ have exceeded $U_{1}$; that is, $(i-1) \tau<T_{L_{1}} \leq T_{U_{1}}<i \tau . T_{\xi}(i)=i \tau-T_{U_{1}}$ represents the time from the moment when the amount of CO2 emitted exceeds the critical level to the instant of the inspection. Express this event as

$$
\begin{aligned}
C_{1} & (i, \tau) \\
\quad & =\left\{(i-1) \tau<T_{L_{1}} \leq T_{U_{1}}<i \tau \mid T_{\xi}(i)=i \tau-T_{U_{1}}\right\} .
\end{aligned}
$$

Then the expected time for paying a penalty is given by

$$
\begin{aligned}
& E\left[T_{\xi}\right]=\sum_{i=1}^{\infty} P\left\{(i-1) \tau<T_{L_{1}} \leq T_{U_{1}}<i \tau \mid T_{\xi}(i)=i \tau-T_{U_{1}}\right\}=\sum_{i=1}^{\infty} \int_{(i-1) \tau}^{i \tau} \int_{0}^{z-u} \int_{(i-1) \tau}^{z} F(u, s) \cdot(i \tau-z) d u d s d z \\
& \quad=\sum_{i=1}^{\infty} \int_{(i-1) \tau}^{i \tau} \int_{0}^{z-u} \int_{(i-1) \tau}^{z} f_{T_{L_{1}}}(u) f_{T_{U_{1}}-T_{L_{1}}}(s) \cdot(i \tau-z) d u d s d z=\sum_{i=1}^{\infty} \int_{(i-1) \tau}^{i \tau}(i \tau-z) \int_{(i-1) \tau}^{z} f_{T_{L_{1}}}(u) F_{T_{U_{1}}-T_{L_{1}}}(z-u) d u d z
\end{aligned}
$$




$$
\begin{aligned}
& =\sum_{i=1}^{\infty} \int_{(i-1) \tau}^{i \tau}(i \tau-z) \int_{(i-1) \tau}^{z} \frac{L_{1}}{\sqrt{2 \pi u^{3} \sigma_{1}^{2}}} \exp \left(-\frac{\left(L_{1}-\lambda_{1} u\right)^{2}}{2 \sigma_{1}^{2} u}\right) \\
& \cdot\left[\Phi\left(\frac{-\left(U_{1}-L_{1}\right)+\lambda_{1} u}{\sigma_{1} \sqrt{u}}\right)+\exp \left(\frac{2 \lambda_{1}\left(U_{1}-L_{1}\right)}{\sigma_{1}^{2}}\right) \Phi\left(\frac{-\left(U_{1}-L_{1}\right)-\lambda_{1} u}{\sigma_{1} \sqrt{u}}\right)\right] d u d z .
\end{aligned}
$$

(b) The Average Time for Excess Amount of Energy Consumption. At $i \tau$, the energy consumption $X_{2}(t)$ has exceeded $U_{2}$; that is, $(i-1) \tau<T_{L_{2}} \leq T_{U_{2}}<i \tau . T_{\delta}(i)=i \tau-T_{U_{2}}$ represents the average time between the instant when the amount of energy consumption exceeds the critical level and the moment of the inspection. Express this event as

$$
\begin{aligned}
& C_{2}(i, \tau) \\
& \quad=\left\{(i-1) \tau<T_{L_{2}} \leq T_{U_{2}}<i \tau \mid T_{\delta}(i)=i \tau-T_{U_{2}}\right\} .
\end{aligned}
$$

So the expected time of paying a penalty is given by

$$
\begin{aligned}
E & {\left[T_{\delta}\right]=\sum_{i=1}^{\infty} P\left\{(i-1) \tau<T_{L_{2}} \leq T_{U_{2}}<i \tau \mid T_{\delta}(i)=i \tau-T_{U_{2}}\right\}=\sum_{i=1}^{\infty} \int_{(i-1) \tau}^{i \tau} \int_{0}^{w-v} \int_{(i-1) \tau}^{w} F(v, t) \cdot(i \tau-w) } \\
& =\sum_{i=1}^{\infty} \int_{(i-1) \tau}^{i \tau} \int_{0}^{w-v} \int_{(i-1) \tau}^{w} f_{T_{L_{2}}}(v) f_{T_{U_{2}}-T_{L_{2}}}(t) \cdot(i \tau-w) d v d t d w=\sum_{i=1}^{\infty} \int_{(i-1) \tau}^{i \tau}(i \tau-w) \\
& \cdot \int_{(i-1) \tau}^{w} f_{T_{L_{2}}}(v) F_{T_{U_{2}}-T_{L_{2}}}(w-v) d v d w=\sum_{i=1}^{\infty} \int_{(i-1) \tau}^{i \tau}(i \tau-w) \int_{(i-1) \tau}^{w} \frac{L_{2}}{\sqrt{2 \pi u^{3} \sigma_{2}^{2}}} \exp \left(-\frac{\left(L_{2}-\lambda_{2} v\right)^{2}}{2 \sigma_{2}^{2} v}\right) \\
& \cdot\left[\Phi\left(\frac{-\left(U_{2}-L_{2}\right)+\lambda_{2} v}{\sigma_{2} \sqrt{v}}\right)+\exp \left(\frac{2 \lambda_{2}\left(U_{2}-L_{2}\right)}{\sigma_{2}^{2}}\right) \Phi\left(\frac{-\left(U_{2}-L_{2}\right)-\lambda_{2} v}{\sigma_{2} \sqrt{v}}\right)\right] d v d w .
\end{aligned}
$$

3.2.3. Expected Average Cost per Time Unit during the Renewal Cycle. Based on the formula above, the expected average cost per time unit during the renewal cycle is given by

$$
\begin{aligned}
& E C\left(L_{1}, L_{2}, \tau\right)=\frac{E\left[C_{T}\left(L_{1}, L_{2}, \tau\right)\right]}{E\left[C_{\text {cycle }}\left(L_{1}, L_{2}, \tau\right)\right]} \\
& =\frac{C_{C} P_{P}+C_{P} P_{P}+C_{I} E[N]+C_{\mathrm{CO}_{2}} E\left[T_{\xi}\right]+C_{\text {energy }} E\left[T_{\delta}\right]}{E\left[C_{\text {cycle }}\left(L_{1}, L_{2}, \tau\right)\right]},
\end{aligned}
$$

where the numerator is expressed as

$$
\begin{array}{r}
C_{P} \sum_{i=1}^{\infty}\left[P\left(B_{1}(i, \tau)\right)+P\left(B_{2}(i, \tau)\right)+P\left(B_{3}(i, \tau)\right)\right]+C_{C}(1 \\
\left.-\sum_{i=1}^{\infty}\left[P\left(B_{1}(i, \tau)\right)+P\left(B_{2}(i, \tau)\right)+P\left(B_{3}(i, \tau)\right)\right]\right)+C_{I} \\
. \frac{\sum_{i=1}^{\infty}\left[\left(P\left(A_{1}(i, \tau)\right)+P\left(A_{2}(i, \tau)\right)+P\left(A_{3}(i, \tau)\right)\right) i \tau\right]}{\tau}
\end{array}
$$

$$
\begin{aligned}
& +C_{\mathrm{CO}_{2}} \sum_{i=1}^{\infty} \int_{(i-1) \tau}^{i \tau}(i \tau-z) \\
& \cdot \int_{(i-1) \tau}^{z} \frac{L_{1}}{\sqrt{2 \pi u^{3} \sigma_{1}^{2}}} \exp \left(-\frac{\left(L_{1}-\lambda_{1} u\right)^{2}}{2 \sigma_{1}^{2} u}\right) \\
& \cdot\left[\Phi\left(\frac{-\left(U_{1}-L_{1}\right)+\lambda_{1} u}{\sigma_{1} \sqrt{u}}\right)+\exp \left(\frac{2 \lambda_{1}\left(U_{1}-L_{1}\right)}{\sigma_{1}^{2}}\right)\right. \\
& \left.\cdot \Phi\left(\frac{-\left(U_{1}-L_{1}\right)-\lambda_{1} u}{\sigma_{1} \sqrt{u}}\right)\right] d u d z \\
& +C_{\text {energy }} \sum_{i=1}^{\infty} \int_{(i-1) \tau}^{i \tau}(i \tau-w) \\
& \cdot \int_{(i-1) \tau}^{w} \frac{L_{2}}{2 \pi u^{3} \sigma_{2}^{2}} \exp \left(-\frac{\left(L_{2}-\lambda_{2} v\right)^{2}}{2 \sigma_{2}^{2} v}\right) \\
& \cdot\left[\Phi\left(\frac{-\left(U_{2}-L_{2}\right)+\lambda_{2} v}{\sigma_{2} \sqrt{v}}\right)+\exp \left(\frac{2 \lambda_{2}\left(U_{2}-L_{2}\right)}{\sigma_{2}^{2}}\right)\right. \\
& \left.\cdot \Phi\left(\frac{-\left(U_{2}-L_{2}\right)-\lambda_{2} v}{\sigma_{2} \sqrt{v}}\right)\right] d v d w .
\end{aligned}
$$

The denominator is given by 


$$
\sum_{i=1}^{\infty}\left[\left(P\left(A_{1}(i, \tau)\right)+P\left(A_{2}(i, \tau)\right)+P\left(A_{3}(i, \tau)\right)\right) i \tau\right]
$$

$$
\begin{aligned}
& \sum_{i=1}^{\infty}\left[\left(\int_{(i-1) \tau}^{i \tau} \frac{L_{1}}{\sqrt{2 \pi u^{3} \sigma_{1}^{2}}} \exp \left(-\frac{\left(L_{1}-\lambda_{1} u\right)^{2}}{2 \sigma_{1}^{2} u}\right) d u \int_{i \tau}^{\infty} \frac{L_{2}}{\sqrt{2 \pi v^{3} \sigma_{2}^{2}}} \exp \left(-\frac{\left(L_{2}-\lambda_{2} v\right)^{2}}{2 \sigma_{2}^{2} v}\right) d v+\int_{i \tau}^{\infty} \frac{L_{1}}{\sqrt{2 \pi u^{3} \sigma_{1}^{2}}} \exp \left(-\frac{\left(L_{1}-\lambda_{1} u\right)^{2}}{2 \sigma_{1}^{2} u}\right) d u \int_{(i-1) \tau}^{i \tau} \frac{L_{2}}{\sqrt{2 \pi v^{3} \sigma_{2}^{2}}} \exp \left(-\frac{\left(L_{2}-\lambda_{2} v\right)^{2}}{2 \sigma_{2}^{2} v}\right) d v\right.\right. \\
& \left.\left.\quad+\int_{(i-1) \tau}^{i \tau} \frac{L_{1}}{\sqrt{2 \pi u^{3} \sigma_{1}^{2}}} \exp \left(-\frac{\left(L_{1}-\lambda_{1} u\right)^{2}}{2 \sigma_{1}^{2} u}\right) d u \int_{(i-1) \tau}^{i \tau} \frac{L_{2}}{\sqrt{2 \pi v^{3} \sigma_{2}^{2}}} \exp \left(-\frac{\left(L_{2}-\lambda_{2} v\right)^{2}}{2 \sigma_{2}^{2} v}\right) d v\right) i \tau\right]
\end{aligned}
$$

3.3. Numerical Procedure. To integrate the calculations illustrated above, after inputting the parameters $\Delta L_{1}, \Delta L_{2}, \Delta \tau$, $T_{u}, C_{C}, C_{P}, C_{i}, C_{\mathrm{CO}_{2}}$, and $C_{\text {energy }}$, the expected average cost in the renewal cycle can be computed through the numerical procedure in Figure 1. follows.

The detailed steps of the algorithm are described as

Step 1. Initialize $L_{1}=0, L_{2}=0, t=0, i=0$ and let $L_{1}=$ $L_{1}+\Delta L_{1}, L_{2}=L_{2}+\Delta L_{2}$, and $t=t+\Delta \tau$.

Step 2. Inspect amount of $\mathrm{CO} 2$ emissions $X_{1}(i t)$ and energy consumption $X_{2}(i t)$ at it.

Step 3. If $X_{1}$ (it) $<L_{1}$ and $X_{2}$ (it) $<L_{2}$, let $i=i+1$ and go to Step 2; otherwise, go to Step 4.

Step 4. If $t<T_{u}$, let $t=t+\Delta \tau$ and go to Step 2; otherwise, go to Step 5.

Step 5. Compute $E C\left(L_{1}, L_{2}\right.$, it $)$ for each it and store the minimum $E C_{1}\left(L_{1}, L_{2}\right.$, it $)$.

Step 6. If $L_{1}<U_{1}$, let $L_{1}=L_{1}+\Delta L_{1}$ and go to Step 2; otherwise, go to Step 7.

Step 7. Compute $E C_{1}\left(L_{1}, L_{2}\right.$,it) for each $L_{1}$ and store the minimum $E C_{2}\left(L_{1}, L_{2}\right.$, it $)$.

Step 8. If $L_{2}<U_{2}$, let $L_{2}=L_{2}+\Delta L_{2}$ and go to Step 2; otherwise, go to Step 9.

Step 9. Compute $E C_{2}\left(L_{1}, L_{2}\right.$, it $)$ for each $L_{2}$ and store the minimum $E C_{\min }\left(L_{1}, L_{2}\right.$, it $)$.

Step 10. Output $E C_{\min }\left(L_{1}, L_{2}, i t\right)$ and the corresponding $L_{1}, L_{2}$, it.

\section{Step 11. End.}

The above is the fully unfolded formula for the average expected cost per time unit within the renewal cycle. Next, we use the simulation method to obtain the optimal interval of periodic inspection, the PM threshold level of $\mathrm{CO} 2$ emissions, and energy consumption and then give the most appropriate maintenance strategy. In addition, sensitivity analyses will be conducted on the penalty cost for excess $\mathrm{CO} 2$ emissions, the penalty cost for excess energy consumption, the inspection cost, the PM cost, and the CM cost.

\section{Simulation Analysis and Sensitivity Analysis}

4.1. Basic Assumption of the Model. The model assumes that the critical level of $\mathrm{CO} 2$ emissions and energy consumption fixed by legislation are known constants. The critical level of $\mathrm{CO} 2$ emissions will be set at 10 tons with reference to Tlili et al.s study [12], which are presented in Table 1.

In terms of the threshold of energy consumption, it is important to note that since much equipment now uses mixed fuels to reduce environmental pollution (e.g., the mixed boiler which, apart from coal, also consumes diesel and natural gas), the consumption of a single energy source is not proportional to the overall $\mathrm{CO} 2$ emissions of the system. In other words, energy consumption and $\mathrm{CO} 2$ emissions are independent and thus call for an integrative consideration in this study. Notice each threshold cannot be obtained from the other directly. Here, one energy source will be separated from mixed energy and studied, which is assumed to be coal. Given that China sets different coal limits on various companies, we refer to the coal limit of one specific company as the benchmark and assume the critical level of energy consumption is 4 tons. Again, in reference to Tlili et al.'s study, the critical level of CO2 emissions will be set to 10 tons for the simulation. Other values $\left(\mathrm{PM} \operatorname{cost} C_{P}, \mathrm{CM}\right.$ cost $C_{C}$, inspection $\operatorname{cost} C_{I}$, and penalty cost for excess $\mathrm{CO} 2$ emission $\left.C_{\mathrm{CO}_{2}}, \lambda_{1}, \sigma_{1}\right)$ also refer to Tlili et al.s study.

The pdf of the time from current time to the moment when $\mathrm{CO} 2$ emission reaches the critical value is given by

$$
f_{T_{U_{1}}}(t)=\frac{10}{\sqrt{2 \pi t^{3} 0.35^{2}}} \exp \left(-\frac{(10-1.3 t)^{2}}{2 \cdot 0.35^{2} t}\right) .
$$

Its cdf is expressed as

$$
\begin{aligned}
F_{T_{U_{1}}}(t)= & \Phi\left(\frac{-10+1.3 t}{0.35 \sqrt{t}}\right) \\
& +\exp \left(\frac{2 \cdot 1.3 \cdot 10}{0.35^{2}}\right) \Phi\left(\frac{-10-1.3 t}{0.35 \sqrt{t}}\right) .
\end{aligned}
$$

The pdf of the time from the instant at which $\mathrm{CO} 2$ emissions hit the threshold to the moment when it reaches the critical value is given by

$$
\begin{aligned}
& f_{T_{U_{1}}-T_{L_{1}}}(t) \\
& \quad=\frac{10-L_{1}}{\sqrt{2 \pi t^{3} 0.35^{2}}} \exp \left(-\frac{\left(10-L_{1}-1.3 t\right)^{2}}{2 \cdot 0.35^{2} t}\right) .
\end{aligned}
$$


TABLE 1: Known data.

\begin{tabular}{lcccccccccc}
\hline$C_{C}$ (USD) & $C_{P}$ (USD) & $C_{I}$ (USD) & $C_{\mathrm{CO} 2}$ (USD/week) & $C_{\text {energy }}$ (USD/week) & $U_{1}$ & $U_{2}$ & $\lambda_{1}$ & $\sigma_{1}$ & $\lambda_{2}$ & $\sigma_{2}$ \\
\hline 900 & 500 & 100 & 10000 & 3500 & 10 & 4 & 1.3 & 0.35 & 0.416 & 0.112 \\
\hline
\end{tabular}

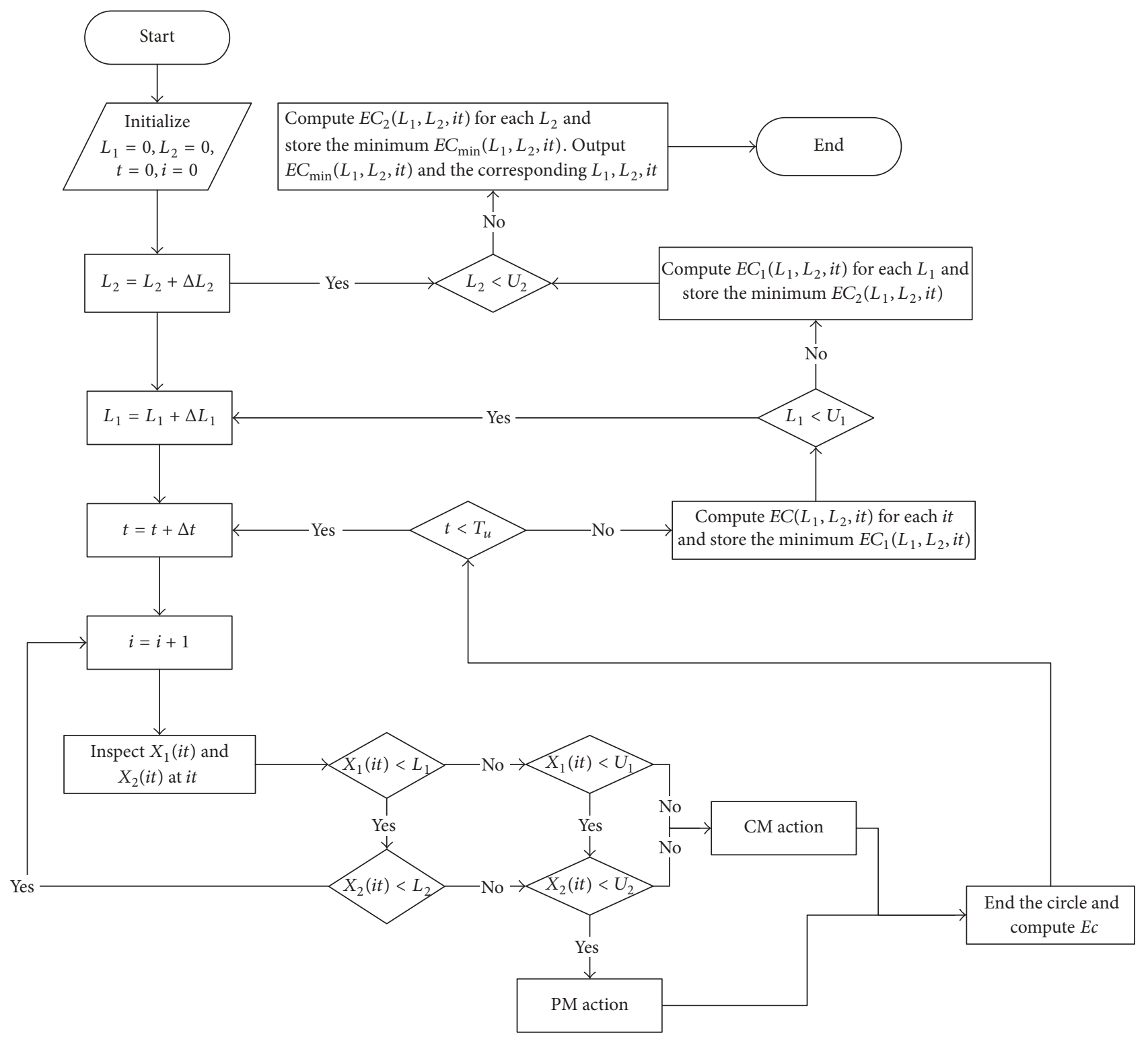

FIGURE 1: The numerical procedure for the expected average cost.

Its cdf is expressed as

$$
\begin{aligned}
F_{T_{U_{1}}-T_{L_{1}}}(t) & \\
= & \Phi\left(\frac{L_{1}-10+1.3 t}{0.35 \sqrt{t}}\right) \\
& +\exp \left(\frac{2 \cdot 1.3\left(10-L_{1}\right)}{0.35^{2}}\right) \Phi\left(\frac{L_{1}-10-1.3 t}{0.35 \sqrt{t}}\right) .
\end{aligned}
$$

Therefore, the pdf of the time from current time to the moment when energy consumption reaches the critical value is given by

$$
f_{T_{U_{2}}}(t)=\frac{4}{\sqrt{2 \pi t^{3} 0.112^{2}}} \exp \left(-\frac{(4-0.416 t)^{2}}{2 \cdot 0.112^{2} t}\right)
$$


Its cdf is expressed as

$$
\begin{aligned}
F_{T_{U_{2}}}(t)= & \Phi\left(\frac{-4+0.416 t}{0.112 \sqrt{t}}\right) \\
& +\exp \left(\frac{2 \cdot 0.416 \cdot 4}{0.112^{2}}\right) \Phi\left(\frac{-4-0.416 t}{0.112 \sqrt{t}}\right) .
\end{aligned}
$$

The pdf of the time from the instant at which energy consumption hits the threshold to the moment when it reaches the critical value is given by

$$
\begin{aligned}
& f_{T_{U_{2}}-T_{L_{2}}}(t) \\
& \quad=\frac{4-L_{2}}{\sqrt{2 \pi t^{3} 0.112^{2}}} \exp \left(-\frac{\left(4-L_{2}-0.416 t\right)^{2}}{2 \cdot 0.112^{2} t}\right) .
\end{aligned}
$$

Its cdf is expressed as

$$
\begin{aligned}
F_{T_{U_{2}}-T_{L_{2}}}(t) & \\
= & \Phi\left(\frac{L_{2}-4+0.416 t}{0.112 \sqrt{t}}\right) \\
& \quad+\exp \left(\frac{0.832\left(4-L_{2}\right)}{0.112^{2}}\right) \Phi\left(\frac{L_{2}-4-0.416 t}{0.112 \sqrt{t}}\right) .
\end{aligned}
$$

4.2. Comparative Analysis. The $\mathrm{CO} 2$ emissions and energy consumption of the system are simulated using the Monte Carlo method and the Particle Swarm Optimization (PSO) algorithm is used to accelerate the operation. The optimal thresholds of $\mathrm{CO} 2$ emissions and energy consumption and the time interval of periodic inspection are obtained by minimizing the expected cost per time unit during the renewal cycle.

Results obtained, which are shown in Table 2, give the optimal threshold of $\mathrm{CO} 2$ emissions as 6 tons and the one of energy consumption as 3 tons and the interval for periodic inspection as 7 week, when the renewal period is 7.002 week. With the limitation of energy consumption, the time of generation of excess CO2 emissions is relatively low $(0.0181$ week), compared with Tlili et al. [12], whose time of generation of it is 0.02 . The average expected cost per week is $\$ 109.406$, which is much lower than the one from Tlili et al. [12], \$123.94. Therefore, the method of this paper performs better in terms of average expected cost.

4.3. Comparative Sensitivity Analysis. This section will vary the penalty cost for excess $\mathrm{CO} 2$ emissions $\mathrm{C}_{\mathrm{CO}_{2}}$, penalty cost for excess energy consumption $C_{\text {energy }}$, inspection cost $C_{I}$, and $C M$ cost $C_{C}$, respectively, compared with the results of Tlili et al.s [12] research, to study their influence on the optimal solution and figure out which method performs better.

4.3.1. Penalty Cost for Excess CO2 Emissions $\mathrm{C}_{\mathrm{CO}_{2}}$. The optimum values are obtained under different circumstances by varying the penalty cost of excess $\mathrm{CO} 2$ emissions. Moreover, the comparison between the results of the paper and Tlili et al. [12] is shown in Table 3 .
First, with the increase of $C_{\mathrm{CO}_{2}}$, its threshold $L_{1}$ decreases and the probability of executing a PM action and the frequency of inspection increases, while the average renewal cycle length shrinks, possibly because the increase in penalty for $\mathrm{CO} 2$ emissions forces companies to tightly monitor the emission of $\mathrm{CO} 2$. Companies try to minimize the possibility that $\mathrm{CO} 2$ emissions exceed the critical level by reducing its threshold along with the interval of periodic inspections, so as to avoid the heavy penalty induced. Moreover, it is worth noticing that, in every pair, the time of generation excess $\mathrm{CO} 2$ emission is less than the Tlili et al.s [12], which may results from the limitation of energy consumption. Furthermore, compared to the method from Tlili et al. [12], in every pair, the expected average cost is much lower.

4.3.2. Penalty Cost for Excess Energy Consumption $C_{\text {energy }}$. The optimum values are obtained under different circumstances by varying the value of the penalty for excessive energy consumption, and the comparison between the results of the paper and Tlili et al. [12] is shown in Table 4.

It can be inferred that, as the penalty cost for excess energy consumption increases, its threshold $L_{2}$ decreases and the probability of executing PM action decreases. It is worth noting that the results keep relatively still in case the penalty of excess energy consumption is 0 and $\$ 3500$. This probably is because when energy consumption reaches its critical level, the $\mathrm{CO} 2$ emissions have already exceeded its critical level, whose induced penalty could counteract the advantage from the reduction of penalty for excess energy consumption. Meanwhile, the expected time of generation of excess $\mathrm{CO} 2$ emissions increases, probably because the penalty of $\$ 35000$ for excess energy consumption weighs way more than the one for excess $\mathrm{CO} 2$ emissions, which makes it worth of taking the cost from the penalty for excess $\mathrm{CO} 2$ emissions. Besides, the table also shows that when the penalty of excess energy consumption is 0 and $\$ 3500$, the average expected cost is relatively lower than the result of Tlili et al. [12]. However, when the penalty increases to $\$ 35000$, the cost increases so rapidly that exceeds the Tlili et al's. We think this is because the penalty of energy consumption contributes too much compared the one of $\mathrm{CO} 2$ emissions.

4.3.3. Inspection Cost $C_{I}$. The optimum values are obtained under different circumstances by varying the value of inspection costs. The comparison between the results of the paper and Tlili et al. [12] is shown in Table 5.

This result shows that, as the inspection cost increases, the threshold of CO2 emissions $L_{2}$ decreases and the interval of periodic inspection increases, whereas the average renewal cycle length increases. Since the company extends the interval of periodic inspection to reduce the total cost, less frequent inspections increase the average time of generation of excess $\mathrm{CO} 2$ emissions and energy consumption; therefore, the penalty cost for excess energy consumption contributes substantially to the average cost of the firm. Moreover, compared to Tlili et al. [12], without the limitation of threshold of energy consumption, the penalty of this paper caused by excess CO2 emissions is much lower than that of Tlili et al.s [12]. Therefore, the average expected cost of this paper is lower 


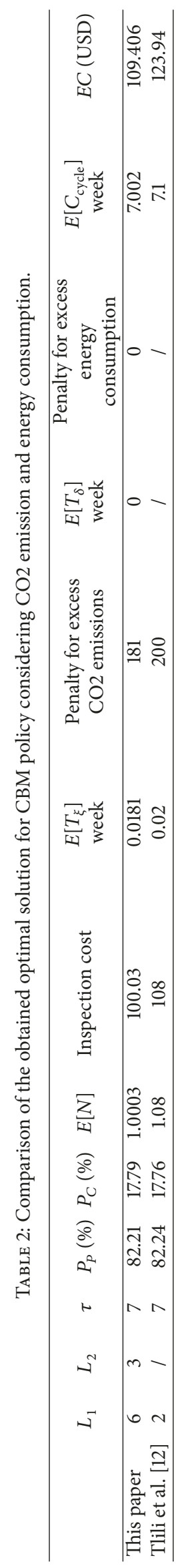




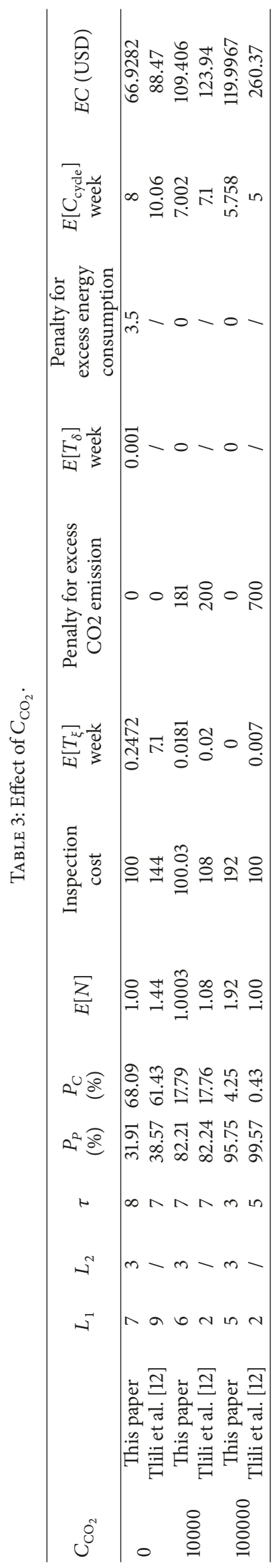




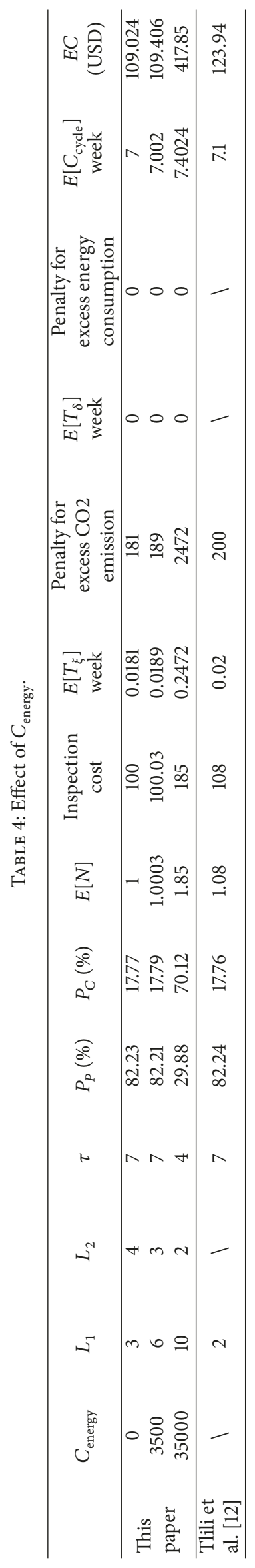




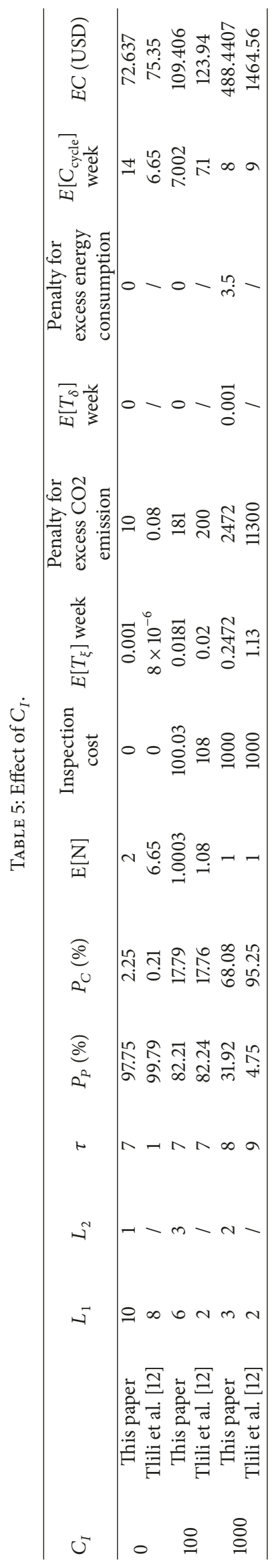


than the one of Tlili et al's [12], especially when the inspection cost increases to $\$ 1000$.

4.3.4. Corrective Maintenance Cost $C_{C}$. The optimum values are obtained under different circumstances by varying the value of the $\mathrm{CM}$ cost. The comparison between the results of the paper and Tlili et al. [12] is shown in Table 6.

The results indicate that as the CM cost increases, the company will lower the two thresholds so as to reduce the probability of executing CM action. Meanwhile, the interval of periodic inspections and the expected average time of excess $\mathrm{CO} 2$ emissions are relatively stable and the inspections keep almost similar frequency, which we think this may be because the balance point for minimizing the expected average cost and its contribution is relative smaller than that of penalty. Since the CM cost increases, as mentioned above, the probability of PM will increase due to its lower cost, which effectively control the time of excess $\mathrm{CO} 2$ emissions and energy consumption. Therefore, the penalty will also decrease, which is lower than the Tlili et al.s [12] results. Thus, this paper's method performs better.

\section{Conclusion and Future Directions}

The original contribution of this paper is to propose a CBM policy for single-unit systems that integrates ecological factors ( $\mathrm{CO} 2$ emissions and energy consumption) into a previous model to obtain the optimal thresholds of $\mathrm{CO} 2$ emission and energy consumption, as well as the interval of periodic inspection, with the objective function of minimizing average expected cost during the cycle. This paper assumes that the system's $\mathrm{CO} 2$ emissions and energy consumption follow two independent normal distributions and that the time from the instant at which one of them hits its corresponding threshold to the moment when it reaches the critical level is independent of the two ecological factors. Based on these two assumptions, the joint pdf of three parameters was established to compute the objective function. We conducted simulations using the Monte Carlo method and algorithm to obtain the optimal threshold of $\mathrm{CO} 2$ emissions, optimal threshold of energy consumption, and the interval of periodic inspections. After a sensitivity study for each variable, there were three discoveries. First, the penalty for excess CO2 emissions and excess energy consumption will affect the values of the company's thresholds. Secondly, the PM costs and CM costs will also influence the values of the two thresholds. Lastly, the inspection cost will affect the time interval of periodic inspections. Meanwhile, the comparison between results whether to take energy consumption or not indicates that only considering $\mathrm{CO} 2$ emissions still leads to some extra penalty, while adding energy consumption effectively reduces the penalty and probability of $\mathrm{CM}$ action, which could result in lower maintenance cost. Therefore, it can be concluded that this paper's method performs better.

This paper leaves much room for further study in two potential directions: (1) to extend CBM policy under periodic inspection to one under nonperiodic inspection; (2) to extend the research object of this article to a multiunit system; and (3) to establish a model of the relationship between $\mathrm{CO} 2$ consumption and mixed energy consumption for a generalized model of optimization of maintenance cost.

\section{Notations}

$\tau$ :

The interval of periodic inspection

$X_{1}(t)$ The Wiener process carbon dioxide

$X_{2}(t): \quad$ The Wiener process energy consumption is subject to

$L_{1}$ : $\quad$ PM threshold level of $\mathrm{CO} 2$ emission

$L_{2}$ : $\quad$ PM threshold level of energy consumption

$U_{1}$ : $\quad$ Critical level of $\mathrm{CO} 2$ emission fixed by legislation

$U_{2}$ : $\quad$ Critical level of energy consumption fixed by legislation

$T_{U}$ : $\quad$ The time $T_{U}$ from present time to the instant one at which the degradation first hits the critical level $U$

$T_{S}$ : $\quad$ The time $T_{S}$ from the moment when the degradation hits the PM threshold $L$ to the instant at which it reaches the critical level $U$

$f_{T_{U}}(t): \quad$ Probability density function associated with $T_{U}$

$F_{T_{U}}(t): \quad$ Cumulative distribution function associated with $T_{U}$

$f_{T_{U}-T_{L}}(t): \quad$ Probability density function associated $T_{S}$

$F_{T_{U}-T_{L}}(t): \quad$ Cumulative distribution associated $T_{S}$

$S:$

The renewal cycle length (A cycle is from current time to the end of maintenance)

$E C\left(L_{1}, L_{2}, \tau\right)$ : The average cycle cost per time unit

$E\left[C_{T}\right]: \quad$ The expected total cost within a cycle

$E\left[C_{\text {cycle }}\right]: \quad$ The expected renewal cycle length

$C_{C}$ :

$C_{P}$ :

$\mathrm{C}_{I}$ :

$\mathrm{C}_{\mathrm{CO}_{2}}$ : Corrective maintenance $(\mathrm{CM})$ action cost Preventive maintenance (PM) action cost Inspection cost emission incurred once the critical level is exceeded

$C_{\text {energy }}$ : Penalty cost per time unit for excess energy consumption incurred once the critical level is exceeded

$P_{\mathrm{C}}$ : $\quad$ Probability that the cycle ends with a CM action

$P_{P}: \quad \quad \quad$ Probability that the cycle ends with a PM action

$E[N]: \quad$ The expected number of inspections during a cycle

$E\left[T_{\xi}\right]: \quad$ The expected average time of generation of excess amount of $\mathrm{CO} 2$ emissions during a cycle

$E\left[T_{\delta}\right]: \quad$ The expected average time of generation of excess amount of energy consumption during a cycle. 


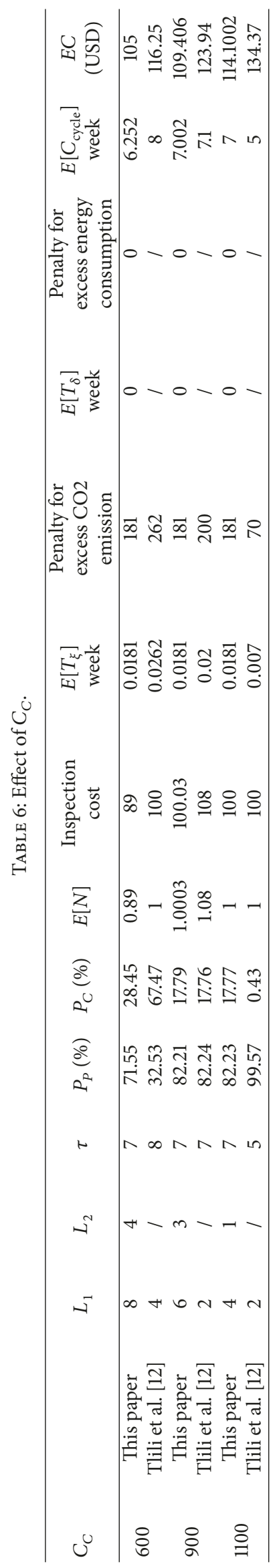




\section{Conflicts of Interest}

The authors declare that there are no conflicts of interest regarding the publication of this paper.

\section{Acknowledgments}

The research described in this paper has been funded by the National Natural Science Foundation of China (Grant no. 71302053).

\section{References}

[1] A. Van Horenbeek, K. Kellens, L. Pintelon, and J. R. Duflou, "Economic and environmental aware maintenance optimization," pp. 343-348.

[2] W. Xu and L. Cao, "Energy efficiency analysis of machine tools with periodic maintenance," International Journal of Production Research, vol. 52, no. 18, pp. 5273-5285, 2014.

[3] M. Shafiee, M. Finkelstein, and C. Bérenguer, "An opportunistic condition-based maintenance policy for offshore wind turbine blades subjected to degradation and environmental shocks," Reliability Engineering \& System Safety, vol. 142, article no. 5310, pp. 463-471, 2015.

[4] M. Mora, J. Vera, C. Rocamora, and R. Abadia, "Energy Efficiency and Maintenance Costs of Pumping Systems for Groundwater Extraction," Water Resources Management, vol. 27, no. 12, pp. 4395-4408, 2013.

[5] M. B. Yildirim and F. G. Nezami, "Integrated maintenance and production planning with energy consumption and minimal repair," The International Journal of Advanced Manufacturing Technology, vol. 74, no. 9-12, pp. 1419-1430, 2014.

[6] A. Hoang, P. Do, and B. Iung, "Energy efficiency performancebased prognostics for aided maintenance decision-making: Application to a manufacturing platform," Journal of Cleaner Production, vol. 142, pp. 2838-2857, 2017.

[7] S. Martorell, J. F. Villanueva, S. Carlos et al., "RAMS+C informed decision-making with application to multi-objective optimization of technical specifications and maintenance using genetic algorithms," Reliability Engineering \& System Safety, vol. 87, no. 1, pp. 65-75, 2005.

[8] C. G. Vassiliadis and E. N. Pistikopoulos, "Maintenance-based strategies for environmental risk minimization in the process industries," Journal of Hazardous Materials, vol. 71, no. 1-3, pp. 481-501, 2000.

[9] H. Chouikhi, A. Khatab, and N. Rezg, "A condition-based maintenance policy for a production system under excessive environmental degradation," Journal of Intelligent Manufacturing, vol. 25, no. 4, pp. 727-737, 2014.

[10] J. Yan and D. Hua, "Energy consumption modeling for machine tools after preventive maintenance," in Proceedings of the IEEE International Conference on Industrial Engineering and Engineering Management (IEEM '10), pp. 2201-2205, IEEE, Macao, China, December 2010.

[11] H. Chouikhi, S. Dellagi, and N. Rezg, "Development and optimisation of a maintenance policy under environmental constraints," International Journal of Production Research, vol. 50, no. 13, pp. 3612-3620, 2012.

[12] L. Tlili, M. Radhoui, and A. Chelbi, "Condition-based maintenance strategy for production systems generating environmental damage," Mathematical Problems in Engineering, vol. 2015, Article ID 494162, 12 pages, 2015. 


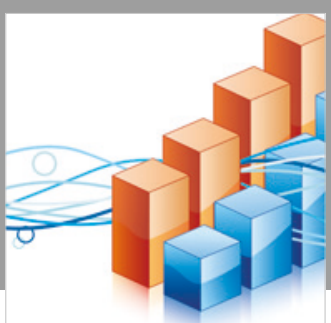

Advances in

Operations Research

\section{-n-m}
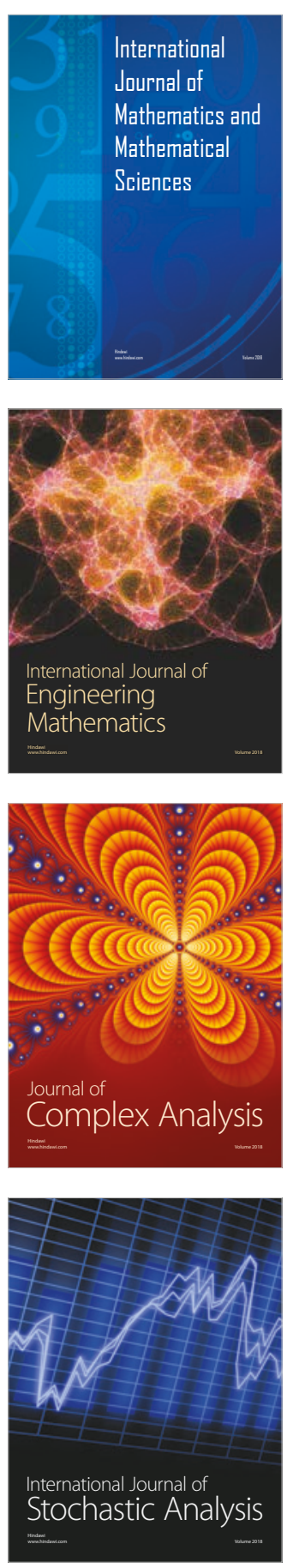
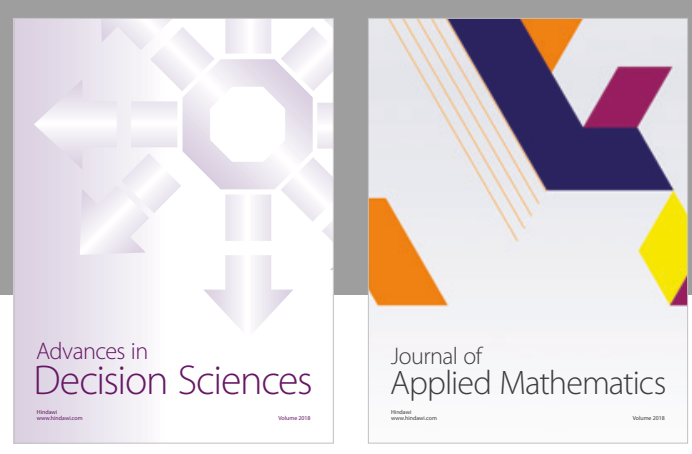

Journal of

Applied Mathematics
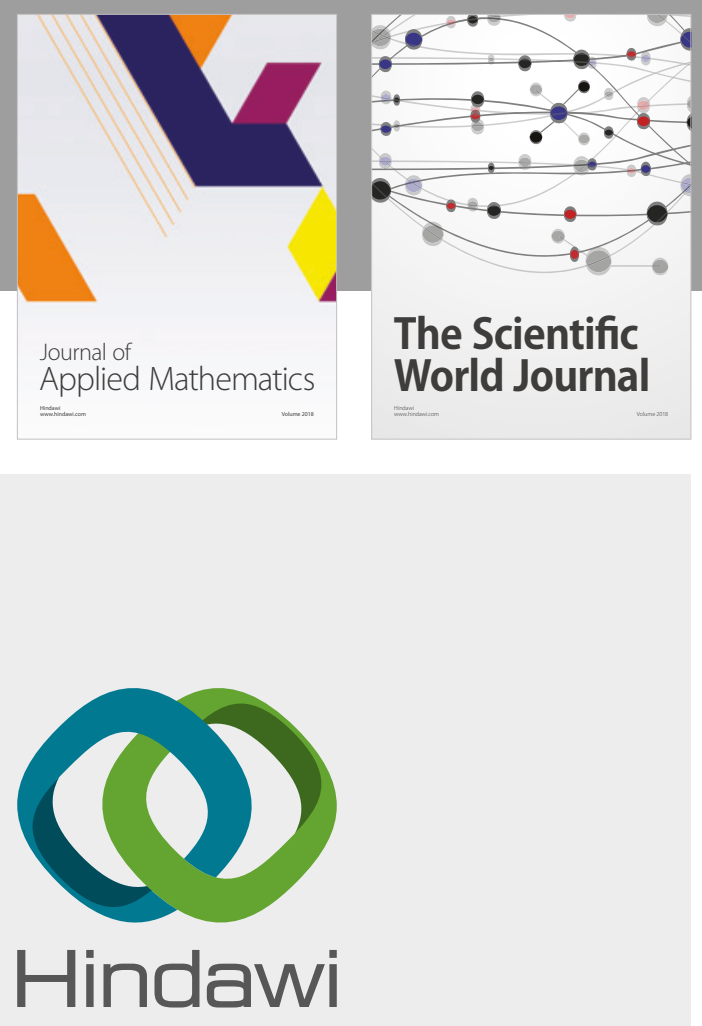

Submit your manuscripts at

www.hindawi.com

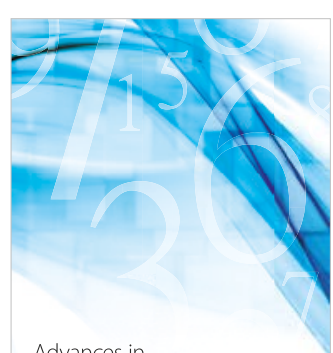

Advances in
Numerical Analysis
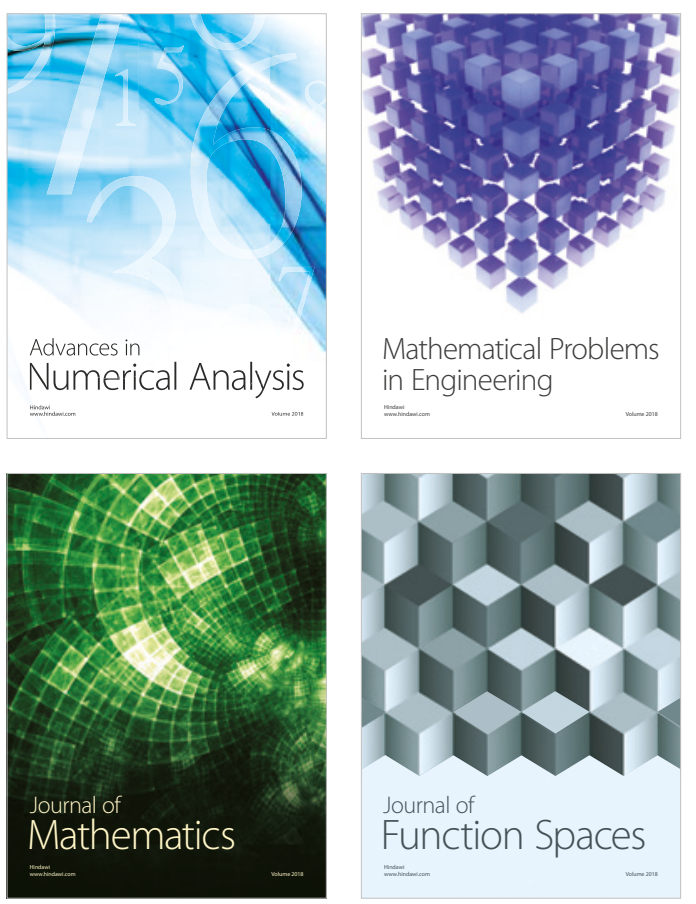

Mathematical Problems in Engineering

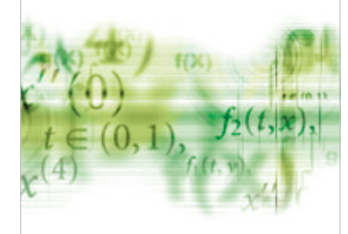

International Journal of

Differential Equations

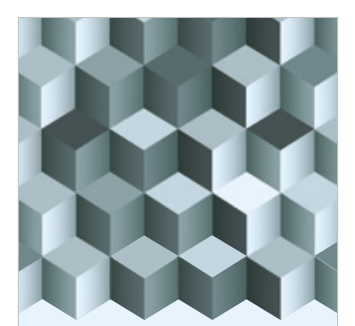

Journal of

Function Spaces

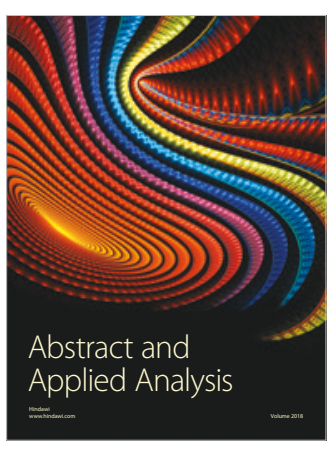

The Scientific

World Journal

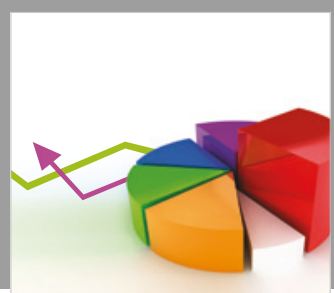

Journal of

Probability and Statistics
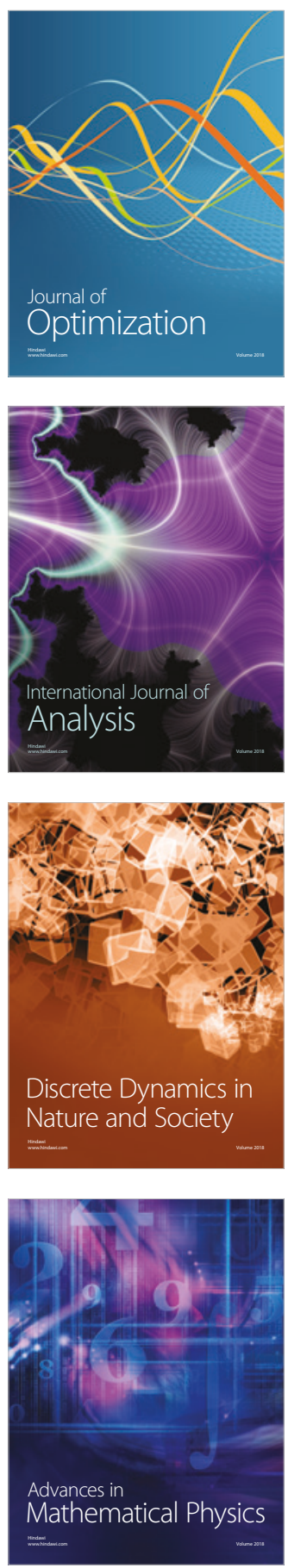